\title{
New or Worsening Symptom
}

National Cancer Institute

\section{Source}

National Cancer Institute. New or Worsening Symptom. NCI Thesaurus. Code C119238.

The subjective evidence of disease perceived by the patient that can be classified as new or worsening 